\title{
Mutation in the First Ig-Like Domain of Kit Leads to JAK2 Activation and Myeloproliferation in Mice
}

\author{
Zan Huang, ${ }^{*}$ Hai-Bin Ruan, ${ }^{*}$ Zeng-Di Zhang, ${ }^{\dagger}$ Weiqian Chen, ${ }^{\star \ddagger \S}$ Zhaoyu Lin, ${ }^{*}$ Hu Zeng, ${ }^{*}$ and Xiang Gao*
}

From the MOE Key Lab of Model Animal for Disease Study,* Model Animal Research Center, Nanjing University, Nanjing; the Nanjing Children's Hospital Affiliated to Nanjing Medical University, ${ }^{\dagger}$ Nanjing; the Institute for Cardiovascular Science, ${ }^{\ddagger}$ Soochow University, Suzhou; and the Department of Cardiovascular Surgery, ${ }^{\S}$ the First Affiliated Hospital of Soochow University, Suzhou, China

\author{
Accepted for publication \\ September 23, 2013. \\ Address reprint requests to \\ Xiang Gao, Ph.D., Model Ani- \\ mal Research Center, Nanjing \\ University, 12 Xuefu Road, \\ Pukou District, Nanjing \\ 210061, China. E-mail: \\ gaoxiang@nju.edu.cn.
}

\begin{abstract}
Myeloproliferative neoplasms constitute a group of hematopoietic neoplasms at the myeloid stem cell level. Although mutations in the receptor tyrosine kinase KIT have been identified in patients with myeloproliferative neoplasm, the functional causality is unknown because of a lack of animal models. Here, we describe a mouse strain harboring a point mutation in the first Ig-like domain of Kit. Intriguingly, the mutant mice develop a myeloproliferative disorder with typical loss-of-function phenotypes in other tissues. The mutant Kit is incompletely $\mathrm{N}$-glycosylated, shows compromised receptor dimerization, and down-regulates Akt and extracellular signal-regulating kinase 1/2 signaling. However, the mutation increases the association of Kit to Janus kinase (JAK)2 and hence the activation of JAK2. The $\beta$ common receptor of the gp140 family interacts and synergizes with Kit to promote JAK2 phosphorylation, which is further enhanced by the Kit mutation. Inhibition of JAK2 suppresses the proliferation of hematopoietic progenitors in vitro and partially rescues myeloproliferation in mice. Our data suggest that overactivation of JAK2 leads to myeloproliferation in Kit mutant mice and provide mechanistic insights for the diagnosis and treatment of myeloproliferative neoplasms in humans. (Am J Pathol 2014, 184: 122-132; http://dx.doi.org/10.1016/j.ajpath.2013.09.019)
\end{abstract}

Myeloproliferative disorders, referred to as myeloproliferative neoplasms (MPNs) by the 2008 World Health Organization classification system, are clonal hematopoietic stem cell proliferative diseases that are characterized by peripheral erythrocytosis, granulocytosis, or thrombocytosis. ${ }^{1}$ It is now well established that MPNs are the result of abnormal tyrosine kinase signal transduction. ${ }^{2,3}$ Classic MPNs include chronic myeloid leukemia (CML), polycythemia vera, essential thrombocythemia, and primary myelofibrosis. CML is defined by the presence of the Philadelphia chromosome and the associated $B C R-A B L$ fusion gene. $J A K 2$ gene mutations (JAK2V617F, JAK2 exon 12 mutations) have been identified in most of the classic Philadelphia chromosome-negative MPNs (polycythemia vera, essential thrombocythemia, and primary myelofibrosis) and some nonclassic $\mathrm{MPNs}^{4-9}$; however, the genetic underpinnings of MPNs that lack $J A K 2$ mutations remain unclear.

Signaling mediated by v-kit Hardy-Zuckerman 4 female sarcoma viral oncogene homolog (KIT), a type III receptor tyrosine kinase, plays pivotal roles in multiple biological processes. Loss-of-function mutations in KIT, which is expressed in hematopoietic progenitor cells, lead to macrocytic anemia. Conversely, activating mutations have been identified in systematic mastocytosis, acute myeloid leukemia, and lymphoma. ${ }^{10}$ Recently, KIT mutations were detected in patients with both $J A K 2 V 617 F$-positive and -negative MPN. ${ }^{1-13}$ It is still unknown whether all these mutations are the functional cause of MPNs.

The ectodomain of KIT contains five Ig-like domains, the first three (D1 to D3) of which are responsible for ligand binding. ${ }^{14,15}$ Crystal structure analysis has identified three surface epitopes separately on D1 to D3 ${ }^{16,17}$ Binding of the ligand stem cell factor (SCF) to the extracellular domain of KIT leads to receptor dimerization, followed by phosphorylation of tyrosine residues and activation of its intrinsic

This work was supported in part by grants 2011CB944104, IRT0835, 2006BAI23B00, 30825024, 30831160513 (X.G.).

Z.H. and H.-B.R. contributed equally to this work.

Current address of H.-B.R., Section of Comparative Medicine, Yale University School of Medicine, New Haven, CT. 
tyrosine kinase activity. When activated, the phosphotyrosine residues interact with a variety of Src homology 2 domain-containing adaptor proteins and elicit multiple downstream pathways such as phosphatidylinositol 3-kinase (PI3-K)/Akt, Ras/extracellular signal-regulated kinase (ERK), and JAK/STAT. ${ }^{18}$

In addition to SCF-induced signaling, KIT physically and functionally interacts with type I cytokine receptors, such as erythropoietin receptor (Epo-R) and IL-7 receptor (IL7R). ${ }^{19-22}$ KIT and receptors for IL-3, granulocyte-macrophage colony-stimulating factor (GM-CSF), and IL-5 also interact and synergize in hematopoietic lineage development. $^{23-25}$ The gp140 common $\beta$ subunit $\left(\beta_{c}\right)$ of IL-3/ GM-CSF/IL-5 receptors may mediate such interaction.

Here, we report a mouse strain, Wps, with a point mutation in the extracellular D1 of Kit that shows a myeloproliferative phenotype, despite the loss-of-function defects in other tissues. Biochemical analyses find that $W p s$ Kit has enhanced physical association with the IL-3/GM-CSF/IL-5 $\beta_{\mathrm{c}}$ receptor and increased JAK2 activation. Therefore, the Wps mouse serves as an ideal animal model of myeloproliferation without $J A K 2$ mutations, which enables us to establish a causal link between Kit mutations and myeloproliferative diseases.

\section{Materials and Methods}

Mice

The $N$-ethyl- $N$-nitrosourea-induced mouse mutagenesis and mutant gene identification were performed as reported previously. ${ }^{26,27}$ Genotyping was performed by PCR, followed by restriction enzyme, AseI digestion. ${ }^{28}$ Littermates from Wps/+ heterozygote crosses were used for all analysis. For fertility assay, age-matched individual wild-type (Wt) or Wps/Wps male mice were mated with two female mice (either genotype) for 1 week and then separated. One week after separation, the females were sacrificed, and the uterine horns were opened. The number of embryos and number of resorptions/stillbirths were counted. If at least one female per mating had live births, the male was considered fertile; if the female had live birth, it was considered fertile.

\section{Hematology Analysis and Progenitor Assay}

Peripheral blood was collected with a capillary tube from suborbital veins and analyzed with a Hemavet multispecies hematology analyzer (Drew Scientific, Dallas, TX). For bone marrow (BM) transplantation, $1 \times 10^{6} \mathrm{BM}$ cells were retrorbitally injected into 8-week-old lethally irradiated recipient mice. Two months later, peripheral blood was collected for hematology and fluorescence-activated cell soring (FACS) analyses. BM cells and spleen cells were plated in MethoCult medium that contained SCF, GM-CSF, IL-3, IL-6, G-CSF, and erythropoietin (StemCell Technologies, Vancouver, BC, Canada) for colony-forming cell assays according to the manufacturer's protocol. AG490, PD98059, and LY294002 were from Beyotime Institute of Biotechnology (Nantong, China) and were dissolved in dimethyl sulfoxide. Two independent assays were performed using three mice for each genotype.

\section{Flow Cytometry}

Flow cytometric analysis was performed with a FACS Calibur system (BD, San Jose, CA). Intracellular staining of Kit (M-14) and phosphor-JAK2 by using indirect conjugated antibodies was performed as described elsewhere. ${ }^{29}$ Kitallophycocyanin (clone 2B8, ACK2), Gr-1-phycoerythrin, Mac1-allophycocyanin antibodies were purchased from eBioscience (San Diego, CA), FITC anti-rabbit IgG was purchased from Sigma-Aldrich (St. Louis, MO), and cyanin 5 anti-goat IgG was purchased from Jackson ImmunoResearch (West Grove, PA).

\section{Plasmids}

Wild-type and Wps Kit cDNA was amplified by three overlapping fragments and ligated sequentially into the EcoRI and HindIII sites of the pcDNA3.1/myc-His(-) A vector (Invitrogen, Carlsbad, CA). The encoded Kit protein has the Myc (alias c$m y c$ ) epitope and polyhistidine tag at the C-terminal. Mouse $\beta_{\mathrm{c}}$ receptor (Csf2rb gene) with Flag tag at the $\mathrm{C}$-terminus followed by stop codon was amplified by PCR and constructed to the XbaI and EcoRI sites of the pcDNA3.1/myc-His(-) A vector. Plasmids with deletions in D1, D1 to D3 of Kit and D1, D4 of $\beta_{\mathrm{c}}$ receptor were produced by a PCR-based method.

\section{Cell Culture, Transfection, and Stable Cell Lines}

Human embryonic kidney (HEK) 293 cells were cultured in Dulbecco's modified Eagle's Medium supplemented with $10 \%$ fetal bovine serum at $37^{\circ} \mathrm{C}$ with $5 \% \mathrm{CO}_{2}$ and transfected with Lipofectamine and PLUS reagent (Invitrogen). For signaling stimulation, cells were serum-deprived overnight then incubated with $100 \mathrm{ng} / \mathrm{mL}$ recombinant SCF (PeproTech, London, UK) for 15 minutes at $37^{\circ} \mathrm{C}$. Cells were lyzed with $1 \%$ Nonidet $\mathrm{P}-40,50 \mathrm{mmol} / \mathrm{L}$ Tris $\cdot \mathrm{HCl}, 0.1$ $\mathrm{mmol} / \mathrm{L}$ EDTA, $150 \mathrm{mmol} / \mathrm{L} \mathrm{NaCl}, 1 \mathrm{mmol} / \mathrm{L}$ dithiothreitol, $0.4 \mathrm{mmol} / \mathrm{L}$ phenylmethylsulfonyl fluoride, $0.1 \mathrm{mmol} / \mathrm{L}$ $\mathrm{Na}_{3} \mathrm{VO}_{4}$, and protease inhibitors. Protein concentration was determined with a BCA Protein Assay Kit (Pierce, Rockford, IL). To establish the Kit-expressing stable cell lines, HEK 293 cells were transfected with Myc-tagged Wt or Wps Kit plasmids for 24 hours, split 1:50 into $10-\mathrm{cm}$ dishes that contained $600 \mu \mathrm{g} / \mathrm{mL} \mathrm{G} 418$, and selected for 20 days. Single clones were amplified, and clones with weak overexpression were chosen for the following experiments.

\section{Radiolabeling and Ligand Binding Assay}

SCF was labeled with ${ }^{125} \mathrm{I}$ (Amersham Bioscience, Buckinghamshire, UK) by using the chloramine $\mathrm{T}$ method. For direct binding analysis, stable cells that expressed Wt or 
Wps Kit in 24-well dishes were washed with PBS and incubated for 90 minutes at room temperature with increasing concentrations of ${ }^{125} \mathrm{I}-\mathrm{SCF}$ in $0.25 \mathrm{~mL}$ of binding buffer (RPMI 1640 medium containing $1 \mathrm{mg} / \mathrm{mL}$ bovine serum albumin and $25 \mathrm{mmol} / \mathrm{L} \mathrm{pH} 7.5$ HEPES). The cells were rinsed four times with ice-cold PBS, solubilized in $1 \mathrm{~mL} /$ well solubilization buffer $(0.1 \mathrm{~N} \mathrm{NaOH}, 0.1 \%$ SDS $)$, and the radioactivity was counted with a $\gamma$-counter.

\section{Antibodies, Immunoprecipitation, and Immunoblotting}

Anti-Kit (M-14), anti-p-Tyr, and anti-Myc antibodies were from Santa Cruz Biotechnology (Santa Cruz, CA); anti-Kit (D13A2), anti-phospho Kit, anti-phospho JAK2, anti-Akt, anti-phospho (S473) Akt, anti-ERK1/2, and anti-phospho ERK1/2 antibodies were from Cell Signaling Technology (Beverly, MA); anti-Flag, anti-rabbit IgG, and anti-goat IgG antibodies were from Sigma-Aldrich; and anti-mouse IgG antibody was from Pierce. For immunoprecipitation, wholecell lysates were mixed with antibodies specified in the text and precipitated by Protein G Sepharose (Amersham Bioscience) beads. Specificity of these antibodies for immunoprecipitation was tested (Supplemental Figure S1). Equal amounts of protein samples were electrophoresed on $8 \%$ SDS-PAGE, transferred to Hybond-P polyminylidene difluoride membrane (Amersham Bioscience), and incubated with appropriate primary antibodies at $4^{\circ} \mathrm{C}$ overnight. Western blot analysis was visualized by peroxidase-conjugated secondary antibodies and electrochemiluminescence substrate (Pierce). For deglycosylation, proteins were treated with Endo $\mathrm{H}_{\mathrm{f}}$ or PNGase F (New England Biolabs, Ipswich, MA) according to the manufacturer's instruction, and then processed for Western blot analysis.

\section{Statistical Analysis}

All data are shown as means \pm SEM and were with the $\chi^{2}$ test, two-tailed Student's $t$-test, or two-way analysis of variance.

\section{Results}

\section{Mutations in the Extracellular D1 of KIT in Human Neoplasms}

Gain-of-function mutations of KIT have been extensively reported in various human cancers. Most of these mutations are located in the transmembrane and the intracellular kinase domains. ${ }^{30}$ Interestingly, there is a relatively high incidence of mutations in the first extracellular Ig-like domain (D1, amino acids 32 to 123) of KIT. We summarize these mutations in Table $1 .{ }^{31}$ Of note, the mutation of aspartic acid to asparagine at amino acid 52 (D52N) has four incidences with three of them in primary myelofibrosis (PML) and CML, both of which are MPNs. However, the roles of these mutations have not yet been explored.

\section{Characterization of $\mathrm{Kit}^{\mathrm{D6ON}}$ Mutant Mice}

Previously, we identified a mouse strain from $N$-ethyl- $N$ nitrosourea-induced mutagenesis that carry a $D 60 N$ mutation in the D1 of Kit. ${ }^{28}$ It serves as an ideal model to study the function of D1 and its potential role in neoplasm development. The strain was designated as Wps according to its primary defects in homozygous mice: $w$ hite, $p$ anda-like, and sub-fertile (Figure 1). Wps/+ heterozygous mice have asymmetric white spots on the belly, and Wps/Wps homozygotes are white with black eyes, black ears, and sometimes black rumps (Figure 1, $\mathrm{A}$ and $\mathrm{B}$ ). Both male and female $W p s / W p s$ mice are viable but show a significant reduction in fertility rate and litter size compared with Wt controls (Figure 1, C and D). The subfertility is due to defects in germ cell development (Figure 1E) (data not shown). We also observed reduced number of skin mast cells in Wps/Wps mice (Figure 1, F and G). These phenotypes suggest $W p s$ as a loss-of-function mutation. ${ }^{27}$

\section{Myeloproliferative Phenotypes in Wps/Wps mice}

Mice that carry loss-of-function mutants in Kit typically have macrocytic anemia. ${ }^{27}$ In contrast, we observed a

Table 1 Mutations in Extracellular D1 of KIT in Human Neoplasms

\begin{tabular}{lllll}
\hline Mutation ID & Mutation (CDS) & Mutation (AA) & Count & Neoplasm \\
\hline COSM268315 & $109 \mathrm{C}>\mathrm{G}$ & P37A & 1 & Large intestine carcinoma \\
COSM447949 & $117 \mathrm{C}>\mathrm{G}$ & I39M & 1 & Breast carcinoma \\
COSM351040 & $146 \mathrm{G}>\mathrm{C}$ & R49P & 1 & Lung carcinoma \\
COSM42898 & $148 \mathrm{G}>\mathrm{A}$ & V50M & 2 & Brain glioma $(n=1)$, large intestine carcinoma $(n=1)$ \\
COSM1146 & $154 \mathrm{G}>\mathrm{A}$ & D52N & 4 & Primary myelofibrosis $(n=2)$, Chronic myeloid leukemia \\
& & & & $(n=1)$, endometrioid carcinoma $(n=1)$ \\
COSM22225 & $217 \mathrm{G}>\mathrm{A}$ & E73K & 1 & Germ cell tumor \\
COSM22226 & $287 \mathrm{C}>\mathrm{T}$ & T96M & 1 & Germ cell tumor \\
COSM139519 & $304 \mathrm{G}>\mathrm{T}$ & $\mathrm{G} 102 \mathrm{C}$ & 1 & Skin carcinoma \\
COSM123991 & $358 \mathrm{G}>\mathrm{T}$ & V120F & 1 & Upper aerodigestive tract carcinoma \\
COSM317523 & $368 \mathrm{C}>\mathrm{T}$ & S123F & 1 & Lung carcinoma \\
\hline
\end{tabular}

All the mutation information was retrieved from the Catalogue of Somatic Mutations in Cancer (COSMIC) of Sanger Institute (http://cancer.sanger.ac.uk/ cancergenome/projects/cosmic, last accessed November 27, 2013).

$A A$, amino acid; CDS, coding sequence. 
A

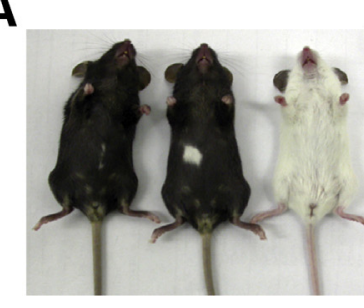

B

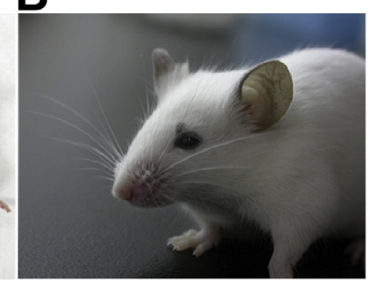

C

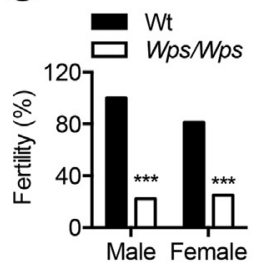

D

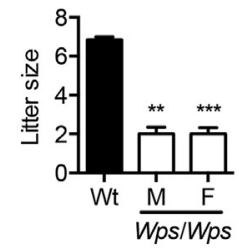

G
$\mathbf{E}$

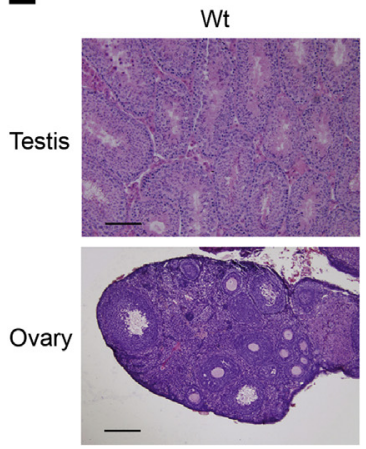

$\mathbf{F}$

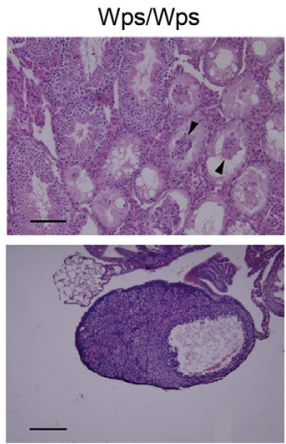

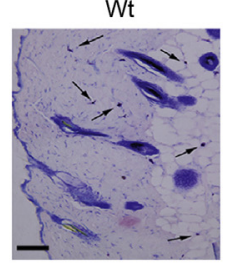
Wps/Wps
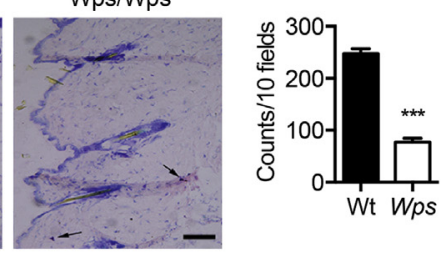

Figure 1 Loss-of-function phenotypes in Wps mice. A: Wps/+ mice (left two mice) with asymmetric white spotting and a Wps/Wps mouse (right mouse). B: Dorsal view of a Wps/Wps mouse showing black eyes and ears. C and D: In vivo fertility assays showing fertility rate (C) and average litter size (D). E: H\&Estained sections of testes and ovaries at the age of 4 months. Wps/Wps male mice have functionally mosaic seminiferous tubules, and females barely have mature follicles. Note clusters of cells indicated by arrowheads and overproliferated Leydig in Wps/Wps testis. F: Skin sections were stained with toluidine blue. Mast cells are in purple, indicated by arrows. G: Total mast cell numbers in 10 objective fields were counted $\left(n=6\right.$ for each genotype). ${ }^{* *} P<0.01$, ${ }^{* * *} P<0.001$ by $\chi^{2}$ test $(\mathbf{C})$ or Student's $t$-test $(\mathbf{D}$ and $\mathbf{G})$. Original magnification: $\times 20(\mathbf{E}) ; \times 10(\mathbf{F})$. Scale bars: $100 \mu \mathrm{m}(\mathbf{E}) ; 200 \mu \mathrm{m}(\mathbf{F})$.

myeloproliferative phenotype with increases in peripheral white blood cell, red blood cell, and platelet counts in both male and female Wps/Wps mice (Figure 2A). Neutrophils, lymphocytes, and monocytes were significantly overproduced, and a slight increase was found in eosinophil and basophil numbers (Figure 2B). The percentages of each white blood cell type were essentially unchanged (data not shown). The profound and consistent decrease in mean corpuscular volume of erythrocytes is totally opposite to that observed in other Kit mutant mice (Figure 2C). ${ }^{27}$ In addition, hemoglobin concentration was higher in female but not male Wps/Wps mice. The mean corpuscular hemoglobin decreased because of the increased red blood cell counts. The mean corpuscular hemoglobin concentration and the red blood cell distributive width were normal in male Wps/Wps mice but increased in female Wps/Wps mice (Supplemental Figure S2A).

To further validate the observed myeloid defects, we stained peripheral blood cells with $\mathrm{Gr}-1$ and Mac-1, followed by flow cytometric analyses. A significant increase was found in immature granulocytes $\left(\mathrm{Gr}-1^{\text {low }} \mathrm{Mac}-1^{+}\right)$and a slight increase in mature granulocytes $\left(\mathrm{Gr}-1^{\mathrm{hi}} \mathrm{Mac}-1^{+}\right)$in Wps/Wps mice, indicating that Wps/Wps mice are myeloproliferative (Figure 2D). We also observed increased infiltration of granulocytes in Wps/Wps spleen and liver (data not shown).

To test whether the myeloproliferation in $W p s / W p s$ mice results from defects in hematopoietic lineages, we transplanted BM cells from Wps/Wps mice into transgenic mice expressing the green fluorescent protein (GFP) driven by the cytomegalovirus promoter, and vice versa (Supplemental Figure S2C). Leukocytes, especially monocytes, increased their numbers in both $W p s / W p s$ mice that received a transplant with GFP BM $\left[W p s^{(\mathrm{GFP} B M)}\right]$ and GFP mice that received a transplant with Wps/Wps BM $\left[\mathrm{GFP}^{(\text {Wps } \mathrm{BM})}\right]$ compared with control mice $\left[\mathrm{Wt}^{(\mathrm{GFP} B M)}\right]$ (Figure 2E). Erythrocyte number increased, whereas mean corpuscular hemoglobin and mean corpuscular volume decreased in $\mathrm{GFP}^{(\text {Wps BM) }}$ mice (Figure $2 \mathrm{~F}$ ). In addition, a slight reduction was found in mean corpuscular volume in $W p s^{(\mathrm{GFP} B M)}$ mice (Figure $2 \mathrm{~F}$ ). These data indicate that $W p s \mathrm{BM}$ transplantation into $\mathrm{Wt}$ mice is able to recapitulate myeloproliferation in Wps/Wps mice, and the BM niche microenvironment also contributes to the phenotypes.

\section{Overproliferated Hematopoietic Progenitors in Wps/ Wps Mice}

To analyze the hematopoietic progenitor cells directly, BM and spleen cells were plated in methylcellulose supplemented with growth factors to form colonies (Figure 3, A and B). Wps/Wps mice showed an expansion in all types of hematopoietic colonies, including burst-forming unit-erythroid, colony-forming unit (CFU)-granulocyte, CFU-monocyte, CFU-granulocyte monocyte, and CFU-granulocyte, erythroid, macrophage, megakaryocyte (Figure 3, C and D). Even with no cytokines or only SCF, IL-6, or erythropoietin, the numbers of various colonies still increased in Wps/Wps $\mathrm{BM}$, suggesting that the overproliferation of hematopoietic 


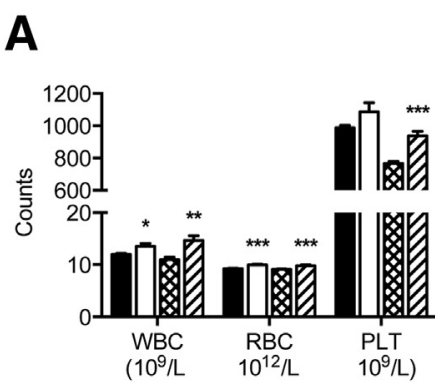

D

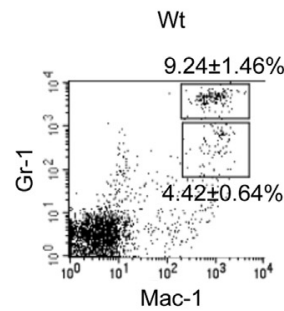

B

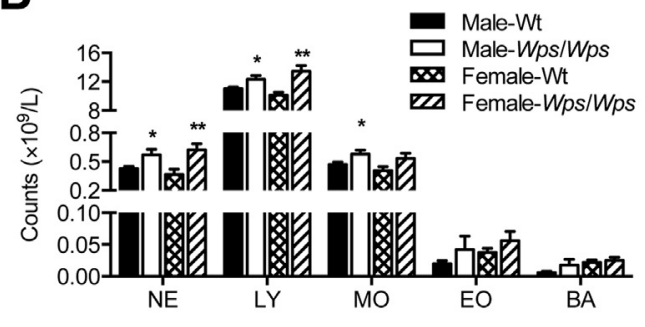

C

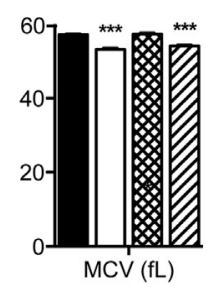

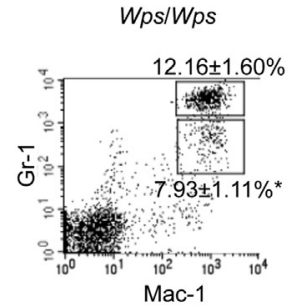

E

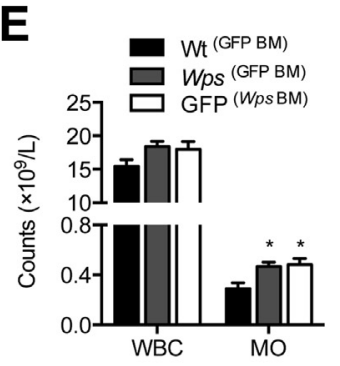

$\mathbf{F}$

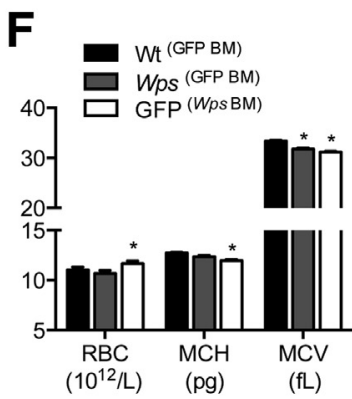

Figure 2 Myeloproliferation in Wps/Wps mice. A-C: Blood from 3-month-old Wt (male, $n=16$; female, $n=16$ ) and Wps/Wps (male, $n=15 ;$ female, $n=$ 14) mice was analyzed. A: Total counts of WBC, RBC, and PLT. B: Counts of WBC subsets. C: MCV values. D: Blood from 6- to 8-week-old male Wt and Wps/Wps mice was stained with Mac-1/Gr-1 and assessed by flow cytometry. Percentages of gated cells are shown. $\mathbf{E}$ and $\mathbf{F}$ : Hematology of peripheral blood from BM mice that received a transplant with $B M$ was analyzed. ${ }^{*} P<0.05,{ }^{*} P<0.01$, and ${ }^{* * *} P<0.001$ by Student's $t$-test $(\mathbf{A}-\mathbf{D})$ and analysis of variance analysis $(\mathbf{E}$ and F). BA, basophil; E0, eosinophil; LY, lymphocyte; MCH, mean corpuscular hemoglobin; MCV, mean corpuscular volume; M0, monocyte; NE, neutrophil; PLT, platelet; RBC, red blood cell; WBC, white blood cell.

progenitors is independent of cytokines (Figure 3E). Histological analysis of the BM indicated that Wps/Wps mice have mild hypercellularity and megakaryocytic hyperplasia with large and hyperlobated nuclei (Figure 3, F and G). These data indicate that overproliferation of hematopoietic progenitors results in myeloproliferation in $W p s / W p s$ mice.

\section{Wps Mutation Blocks the Glycosylation of Kit}

We next determined the biochemical defects in the mutant protein underlying the various phenotypes in Wps/Wps mice. Immunoblotting of Wt Kit that overexpressed in HEK 293 cells displayed two bands with molecular masses of 145 $\mathrm{kDa}$ and $125 \mathrm{kDa}$, respectively, whereas Wps Kit only showed the $125-\mathrm{kDa}$ band (Figure 4A). Kit is a glycoprotein with several consensus glycosylation sites in the extracellular domain. ${ }^{32}$ To test whether the different sizes of Kit are because of different carbohydrate modifications, we treated cells with Endo $\mathrm{H}_{\mathrm{f}}$, which specifically hydrolyzes high mannose oligosaccharides, and PNGase F, which cleaves almost all $N$-linked glycoproteins. The 125-kDa band of both Wt and Wps Kit is sensitive to both Endo $\mathrm{H}_{\mathrm{f}}$ and PNGase F, suggesting that it is a high mannose glycoform. The 145-kDa Wt Kit can be digested by PNGase F but not by Endo $\mathrm{H}_{\mathrm{f}}$; therefore, it contains complex/hybrid $N$-liked oligosaccharide structures. Completely deglycosylated proteins are approximately $110 \mathrm{kDa}$ in molecular mass.

Kit is also highly glycosylated in some, but not all, tissues in mice. In BM and spleen, the mannose and complex/ hybrid forms of Kit can both be detected in $\mathrm{Wt}$ mice, whereas only the mannose form exists in Wps/Wps mice (Figure 4B). These data suggest that the Wps mutation blocks the complex/hybrid glycosylation from the high mannose glycoform both in vitro and in vivo.

\section{Wps Mutation Decreases Ligand-Binding, Receptor Dimerization, and Akt/ERK Signaling}

The extracellular D1 to D3 domain of Kit is primarily responsible for SCF binding ${ }^{16,17}$; thus, we hypothesized that the Wps mutation affects the ligand-receptor interaction. Indeed, a direct ligand-binding assay showed that there is a slight decrease in SCF binding to Wps Kit $\left(\mathrm{K}_{\mathrm{d}}=10.32\right.$ $\mathrm{nmol} / \mathrm{L})$ compared with Wt Kit $\left(\mathrm{K}_{\mathrm{d}}=8.456 \mathrm{nmol} / \mathrm{L}\right)$ (Figure 4C). Kit receptor dimerization after SCF stimulation was also severely diminished in Wps Kit (Figure 4D and Supplemental Figure S3). Consistently, SCF-induced receptor phosphorylation and downstream Akt and ERK activation decreased with Wps Kit in HEK 293 cells (Figure 5A). The phosphorylation of Akt and ERK was also significantly down-regulated and not responsive to SCF in Wps/Wps BM, spleen, skin, and ovary (Figure 5B and Supplemental Figure S4). SCF/Kit-induced PI3-K/Akt and Ras/ERK signaling was critical for proper development of melanocytes, germ cells, and mast cells. ${ }^{33-35}$ Therefore, we proposed that the compromised Akt and ERK activation is responsible for the defects in melanogenesis, fertility, and mast cell development in Wps/Wps mice. Akt and ERK signaling has been extensively shown to positively regulate proliferation and survival; therefore, it is unlikely that the 
A

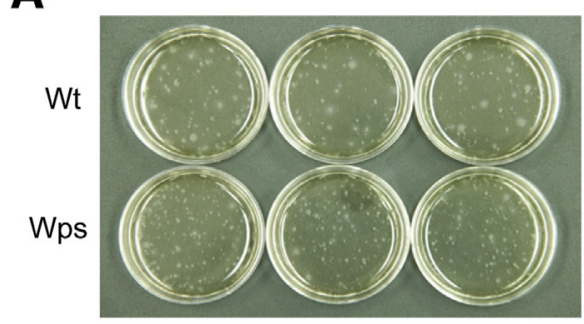

C

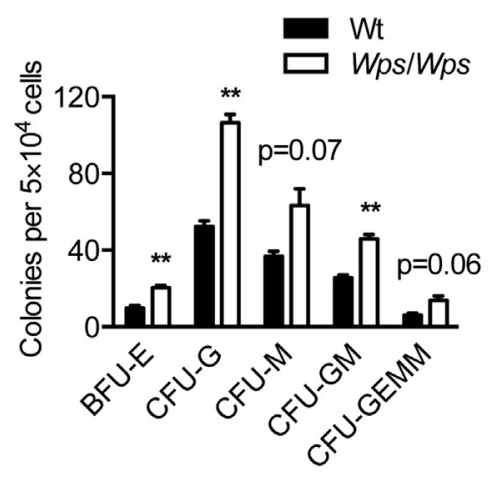

$\mathbf{E}$

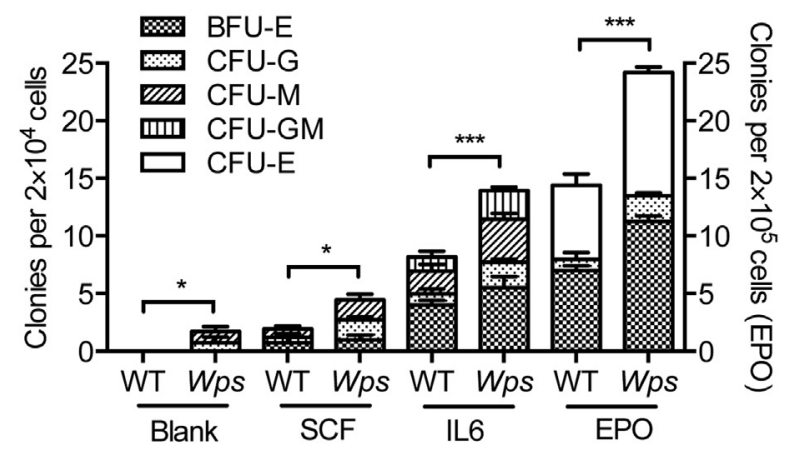

$\mathbf{F}$

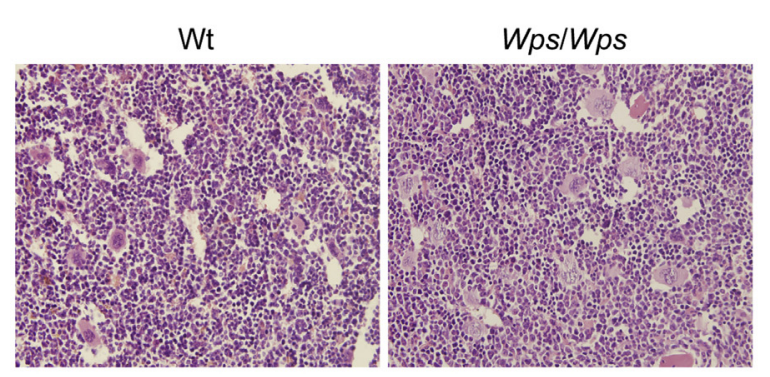

G

deficiency in Akt/ERK signaling leads to myeloproliferation in $W p s / W p s$ mice.

\section{Wps Mutation Enhances JAK2 Signaling}

JAK2 is one downstream target of SCF/Kit signaling, ${ }^{36}$ and activating JAK2 mutations have been identified in patients with MPN. ${ }^{4-6}$ Therefore, we hypothesized that the Wps mutation enhances JAK2 activity and leads to myeloproliferation in mice. As expected, Wps mutation increased the association of JAK2 with Kit, indicated by co-immunoprecipitation experiments in HEK 293 cells (Figure 6A). Furthermore, SCF-induced JAK2

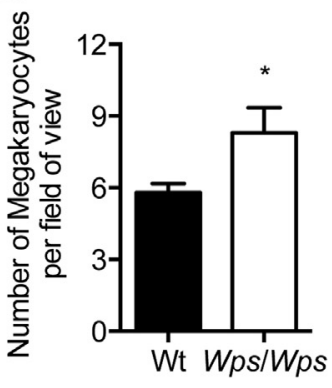

tyrosine phosphorylation was enhanced in the presence of $W p s$ Kit compared with Wt Kit (Figure 6A).

The Wps mutation affected the recognition of the Kit antibodies (clone ACK2 and 2B8, which recognize the extracellular domain of Kit) in both BM cells and transfected HEK 293 cells (Supplemental Figure S5) (data not shown). ${ }^{15}$ Therefore, we performed indirect intracellular staining of freshly isolated BM cells for phospho-JAK2 and Kit. Flow cytometric analyses found increased JAK2 phosphorylation in total (mean fluorescence intensity: $\mathrm{Wt}$, $9.84 \pm 0.41 ;$ Wps/Wps, $12.71 \pm 0.19 ; P<0.01)$ and $\mathrm{Kit}^{+}$ (mean fluorescence intensity: Wt, $5.90 \pm 0.07$; Wps/Wps, 


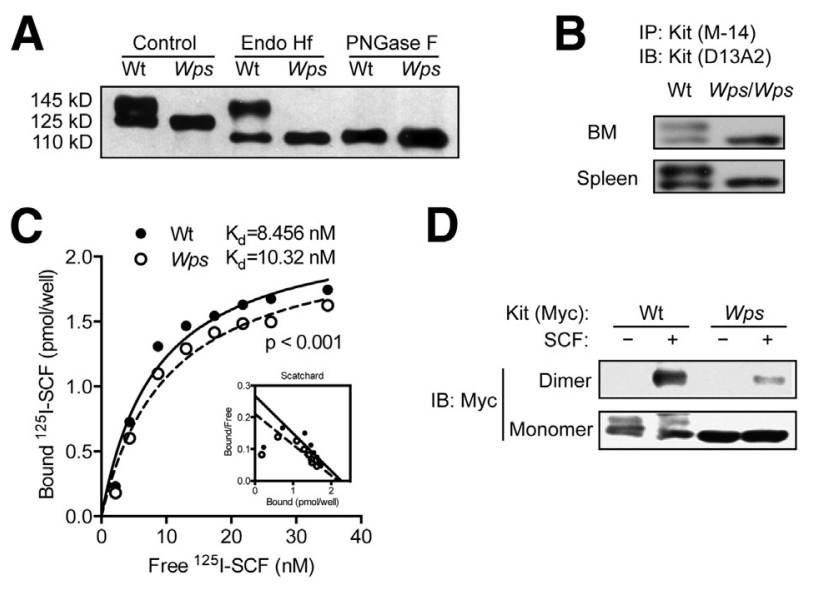

Figure 4 Wps mutation decreases Kit glycosylation, ligand binding, and receptor dimerization. A: Proteins from HEK 293 cells transfected with Wt or Wps Kit were treated with Endo $\mathrm{H}_{\mathrm{f}}$ or PNGase $\mathrm{F}$ and immunoblotted with Myc epitope. The 145-, 125-, and 110-kDa bands indicate complex/hybrid-, high mannose-, and nonglycosylated Kit, respectively. B: Kit expression in Wt and Wps/Wps BM and spleen shown by Kit immunoprecipitation followed by Kit immunoblotting. C: Stable HEK 293 cells expressing Wt or Wps Kit were incubated with different concentrations of ${ }^{125} \mathrm{I}$-SCF. Direct binding data plotted as bound versus free ${ }^{125} \mathrm{I}-\mathrm{SCF}$ are shown. Scatchard plot of the data are shown as an inset. Triple wells were used for each concentration, and one representative data of three independent experiments are shown. $P<0.001$ by two-way analysis of variance. D: Wt or Wps Kit- transfected HEK 293 cells were stimulated with SCF and then cross-linked with $1 \mathrm{mmol} / \mathrm{L}$ bis(sulfosuccinimidyl)suberate (BS3). Monomers and dimers of Kit are identified by their molecular weight. See Supplemental Figure S3 for the whole blot. IB, immunoblotting; IP, immunoprecipitation.

$6.93 \pm 0.06 ; P<0.001$ ) Wps/Wps BM cells (Figure 6B). Western blot analysis confirmed that JAK2 phosphorylation increased in BM but not other tissues (Supplemental Figure S6). These data suggest that Wps Kit promotes JAK2 association and activation both in vitro and in BM.
To validate whether JAK2 overactivation is responsible for the myeloproliferation in Wps/Wps mice, we performed colony formation assays of $\mathrm{BM}$ cells in the presence of inhibitors to different pathways (Figure 6C). JAK2 inhibition (AG490) arrested colony formation of Wps/Wps BM to the level of Wt cells. The mitogen-activated protein extracellular signal-related kinase $1 / 2$ inhibitor had no effect on either Wt or Wps/Wps cells, whereas inhibition of the PI3-K pathway suppressed colony formation in both Wt and Wps/ Wps cells. These data indicate that PI3-K/Akt signaling is important for myeloid development, but only enhanced JAK2 activation is responsible for the overproliferation of progenitor cells in the mutant BM. More importantly, treating Wps/Wps mice with AG490 in vivo for only 15 days reduced the levels of erythrocytes, hematocrit, and hemoglobin (Figure 6D), suggesting that JAK2 inhibition could be effective in treating MPNs in humans.

\section{Wps Kit Tightly Associates with $\beta_{c}$ Receptor}

It is known that IL-3/GM-CSF/IL-5 receptors interact with Kit during hematopoietic development. In addition, $\beta_{c}$ receptor with constitutively active extracellular mutations functionally cross-talks with Epo-R to activate JAK2 ${ }^{37}$, which leads to MPN in mice. ${ }^{38}$ These findings led us to test if the $\beta_{\mathrm{c}}$ receptor cooperates with Wps Kit to activate JAK2.

Co-immunoprecipitation in HEK 293 cells indicated that the Wps mutation enhances the physical interaction between Kit and the $\beta_{\mathrm{c}}$ receptor (Figure 7A). Moreover, overexpression of the $\beta_{\mathrm{c}}$ receptor promotes JAK2/Kit association, Kit receptor autophosphorylation, and JAK2 activation (Figure 7B). JAK2 association and activation were greater in the Wps mutant, despite the deficiency in autophosphorylation of Kit, Akt, and ERK signaling (Figure 7B and

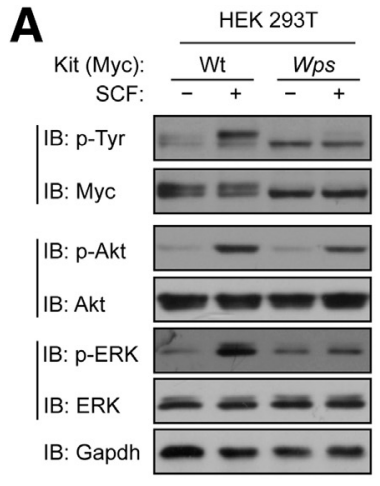

B

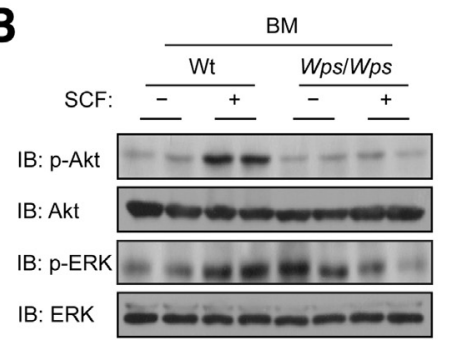

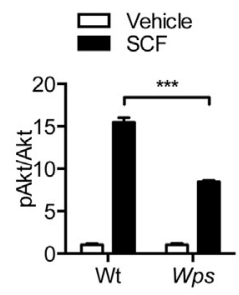
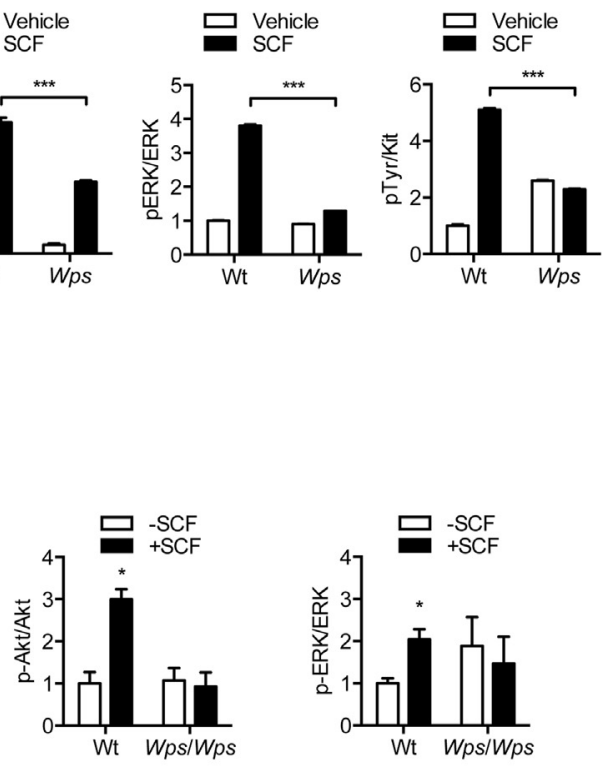

Figure 5 Wps mutation decreases Akt and ERK signaling. A: HEK 293 cells were transfected with Wt or Wps Kit and stimulated with or without $100 \mathrm{ng} / \mathrm{mL}$ SCF for 15 minutes. Kit phosphorylation, activation of Akt and ERK were determined by immunoblotting. Densitometry of three independent experiments is shown in graphs to the right. B: Wt and Wps/Wps mice were intraperitoneally injected with $15 \mathrm{ng}$ of SCF for 30 minutes, and phospho-Akt and phospho-ERK in BM were determined. Densitometric analysis is shown in graphs to the right. ${ }^{*} P<0.05,{ }^{* * *} P<0.001$ by Student's $t$-test. Gapdh, glyceraldehyde phosphate dehydrogenase; IB, immunoblotting; IP, immunoprecipitation. 
Supplemental Figure S7). Taken together, these data indicate that increased JAK2 signaling, probably with the cooperation of the $\beta_{\mathrm{c}}$ receptor, may contribute to myeloproliferation in Wps/Wps mice.

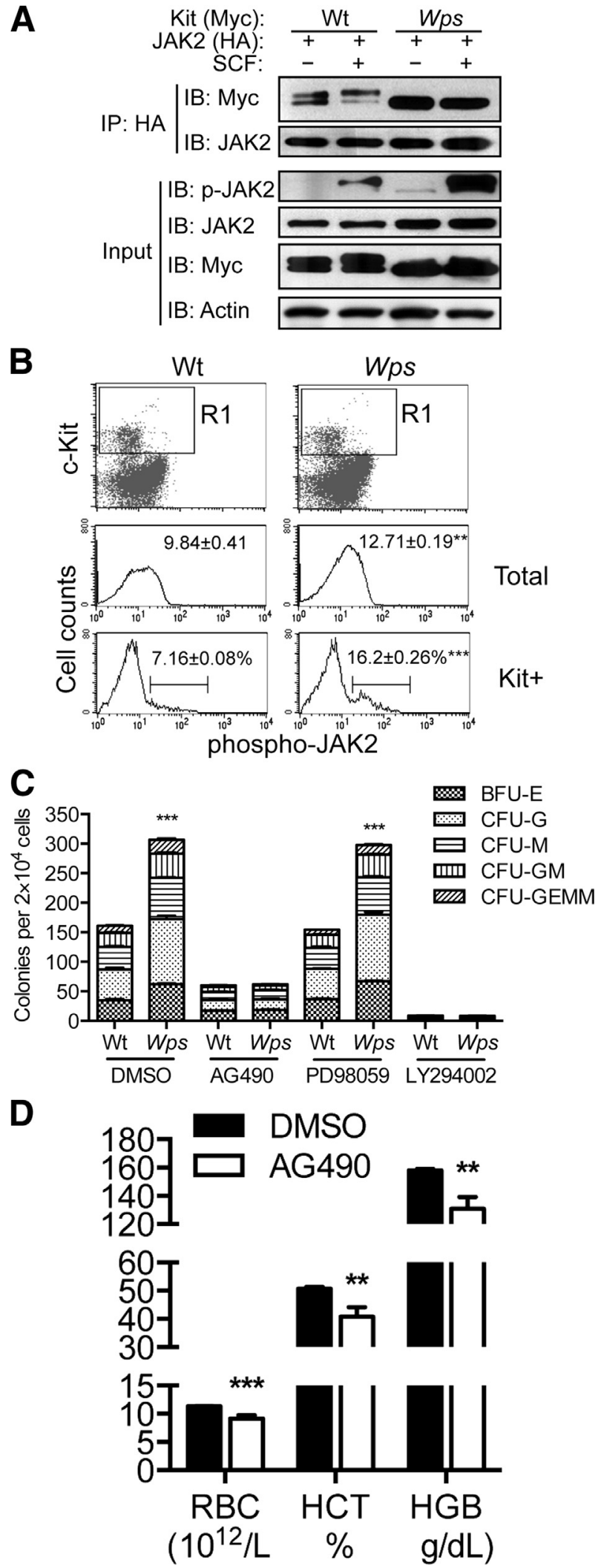

The First Extracellular Domains of Kit and $\beta_{c}$ Receptor Determine Their Interaction

We then asked which domains of Kit and the $\beta_{\mathrm{c}}$ receptor are required for their interaction. Reciprocal co-immunoprecipitation experiments show that deletions of D1 $(\Delta \mathrm{D} 1)$ or D1 to D3 ( $\Delta$ D1-3) of Kit increase the physical association between Kit and the $\beta_{\mathrm{c}}$ receptor (Figure $8 \mathrm{~A}$ ). Binding of the $\beta_{\mathrm{c}}$ receptor to Wps Kit is comparable with the $\Delta \mathrm{D} 1$ and $\Delta$ D1-3 mutants, suggesting that the D1 of Kit inhibits the interaction with the $\beta_{\mathrm{c}}$ receptor.

The extracellular domain of the $\beta_{\mathrm{c}}$ receptor consists of four cytokine receptor domains (D1 to D4), with mutations in D4 conveying constitutive activation. Therefore, we tested the interaction of $\Delta \mathrm{D} 1$ and $\Delta \mathrm{D} 4$ of the $\beta_{\mathrm{c}}$ receptor with Wt Kit (Figure 8B). Only deletion of the D1, but not the $\mathrm{D} 4$, decreased the binding to Kit, indicating that the first extracellular domain of the $\beta_{\mathrm{c}}$ receptor is required for the interaction.

\section{Discussion}

Two types of Kit mutations have been reported in humans and animal models. Loss-of-function mutations cause defects in melanocytes, mast cells, germ cells, and hematogenic cells. In contrast, gain-of-function mutations are known as the cause of mastocytosis, acute myeloid leukemia, gastrointestinal stromal tumors, and germ cell tumors. ${ }^{10}$ The Wps/ $W p s$ mice show deficiencies in melanocytes, germ cells, and mast cells but hyperplasia in myeloid cells. The Wps represents an exclusive example of a Kit mutation with contrary effects in different systems. We also observed decreased Akt and ERK signaling but increased JAK2 activation in Wps mutants. Considering the distinct roles of these pathways in different systems, ${ }^{2,33-35}$ we speculate that the defective Akt and ERK signaling is responsible for the deficiencies in melanogenesis, gametogenesis, and mast cell development,

Figure 6 Enhanced JAK2 association and activation in Wps Kit. Myctagged Kit was co-transfected with HA-tagged JAK2 in HEK 293 cells, followed by SCF stimulation. A: JAK2 and Kit interaction is determined by co-immunoprecipitation. SCF induced JAK2 phosphorylation is shown at the bottom. B: BM cells were isolated, indirectly stained with Kit (M-14) and phospho-JAK2 antibodies, and analyzed by flow cytometry (top). The phospho-JAK2 histograms of the total (no gate) and $\mathrm{Kit}^{+}$(gate R1) cells are shown in the middle and bottom, respectively. Mean fluorescence intensity of total cells and percentage of phospho-JAK2 cells in $\mathrm{Kit}^{+}$population are shown. C: BM cells were plated in methycellulose with growth factors in the presence of DMS0 or $25 \mu \mathrm{mol} / \mathrm{L}$ inhibitor to JAK2 (AG409), 30 $\mu \mathrm{mol} / \mathrm{L}$ ERK1/2 (PD98059), or $25 \mu \mathrm{mol} / \mathrm{L}$ PI3-K (LY294002), and then different colonies were counted $(n=3)$. D: Hematology of Wps/Wps mice treated with two doses of $0.5 \mathrm{mg}$ of AG490 per mouse for 15 days. ${ }^{* *} P<$ $0.01,{ }^{* *} P<0.001$ by Student's $t$-test (B and $\mathbf{D}$ ) and two-way analysis of variance (C). BFU-E, burst-forming unit-erythroid; CFU, colony-forming unit; CFU-G, CFU-granulocyte; CFU-M, CFU-monocyte; CFU-GM, CFU-granulocyte monocyte; CFU-GEMM, CFU-granulocyte, erythroid, macrophage, megakaryocyte; DMSO, dimethyl sulfoxide; HCT, hematocrit; HGB, hemoglobin; IB, immunoblotting; IP, immunoprecipitation; RBC, red blood cell. 


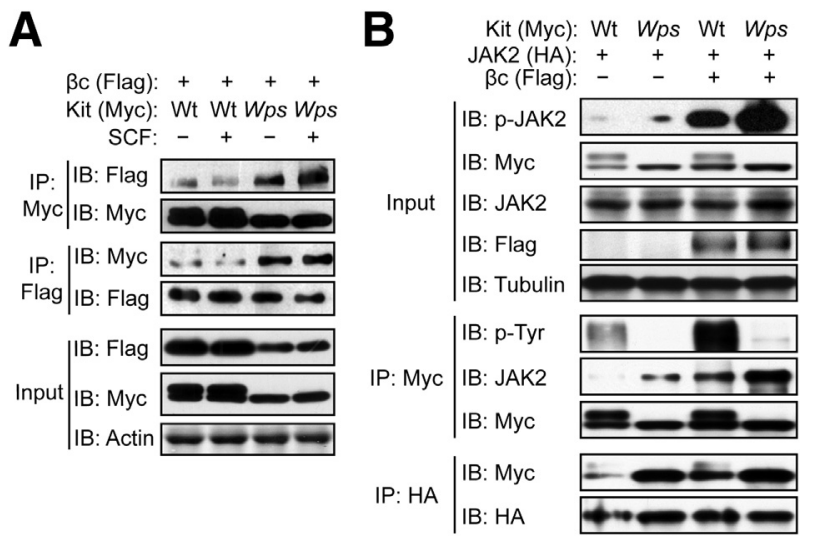

Figure 7 Kit interacts with $\beta_{c}$ receptor. A: Myc-tagged Kit and Flagtagged $\beta_{c}$ receptor were co-expressed in HEK 293 cells. Reciprocal coimmunoprecipitation shows enhanced physical interaction between Wps Kit and $\beta_{c}$ receptor. B: Kit and JAK2 were co-transfected with or without the $\beta_{c}$ receptor in HEK 293 cells. JAK2 phosphorylation, tyrosine phosphorylation of Kit, and protein inputs are shown. IB, immunoblotting; IP, immunoprecipitation.

whereas JAK2 overactivation underlies the myeloproliferation phenotype. However, further investigations are necessary to draw these conclusions. Although mast cells arise from multipotent hematopoietic progenitors in the BM, they mature peripherally within connective or mucosal tissues after leaving the BM. ${ }^{34}$ It is possible that there is also an increase in mast cell progenitors in Wps/Wps BM. However, SCF/Kitmediated Akt and ERK signaling is indispensable for their migration to and survival in peripheral tissues; therefore, skin mast cells decrease their number in $W p s / W p s$ mice.

It is believed that the extracellular D1 to D3 of Kit are the primary binding sites for SCF. The crystal structures of D1 to D3 of mouse Kit and the whole ectodomain of human Kit have been determined. ${ }^{16,17}$ Three SCF/Kit interface surfaces are located in D1, D2, and D3 separately. The interface-I formed by the C' strand (residues 73 to 77 ) in Kit D1 and an extended loop in SCF is considered weak ${ }^{16}$, and D2 to D3 is sufficient for SCF interaction. ${ }^{39,40}$ Consistent with these findings, we only observed a slight decrease in the binding of SCF to Wps Kit (Figure 4C). D4 of Kit is the intrinsic receptor dimerization site, ${ }^{41}$ and we found that the Wps mutation severely dampens receptor dimerization (Figure 4D). It is not likely that the relatively small decrease in ligand binding leads to such dramatic defects in receptor dimer formation and downstream Akt and ERK signaling. The D1 may directly regulate Kit dimerization, independently of its ability to recognize the ligand.

Synergistic effects of IL-3/GM-CSF/IL-5 with SCF have been reported, ${ }^{23,24}$ and constitutively active extracellular mutations in the $\beta_{\mathrm{c}}$ receptor cross-talk with Epo-R to activate JAK $2 .{ }^{37}$ There are direct interactions between Kit and other type I cytokine receptors such as Epo-R and IL$7 R .^{20-22}$ Physical interaction between Kit and the $\beta_{\mathrm{c}}$ receptor has also been reported, ${ }^{25}$ and our study confirms this phenomenon. SCF induces tyrosine phosphorylation of Epo-R and IL-7R in the absence of their ligands, Epo and
IL-3. The same effect is not expected for the $\beta_{\mathrm{c}}$ receptor because the Wps mutation compromises Kit autophosphorylation, whereas the association with the $\beta_{c}$ receptor is enhanced. We propose that a heterodimer of Kit and the $\beta_{\mathrm{c}}$ receptor forms and facilitates the constitutive recruitment of JAK2 to Kit (Figure 8C). The interaction of Kit with JAK2 and the $\beta_{\mathrm{c}}$ receptor does not depend on SCF. The activation of JAK2 depends on SCF stimulation only when the Kit/ $\beta_{c}$ receptor heterodimer is absent. However, if the heterodimer forms, JAK2 will be activated even without treatment of SCF or IL-3. The Wps mutation facilitates $\mathrm{Kit} / \beta_{\mathrm{c}}$ receptor formation and hence overactivates JAK2 signaling. We found that the first extracellular domain of the $\beta_{\mathrm{c}}$ receptor is required for heterodimer formation with Kit. Considering active mutations of the $\beta_{\mathrm{c}}$ receptor lead to MPNs, ${ }^{37,38}$ it would be interesting to determine whether these mutations enhance the interaction with the Kit receptor to induce cytokine-independent proliferation.

Endogenous $\beta_{\mathrm{c}}$ receptor expression in 293 cells is low, and overexpressing it enhances JAK2 association with Kit. We propose that the intrinsic properties of Kit and the

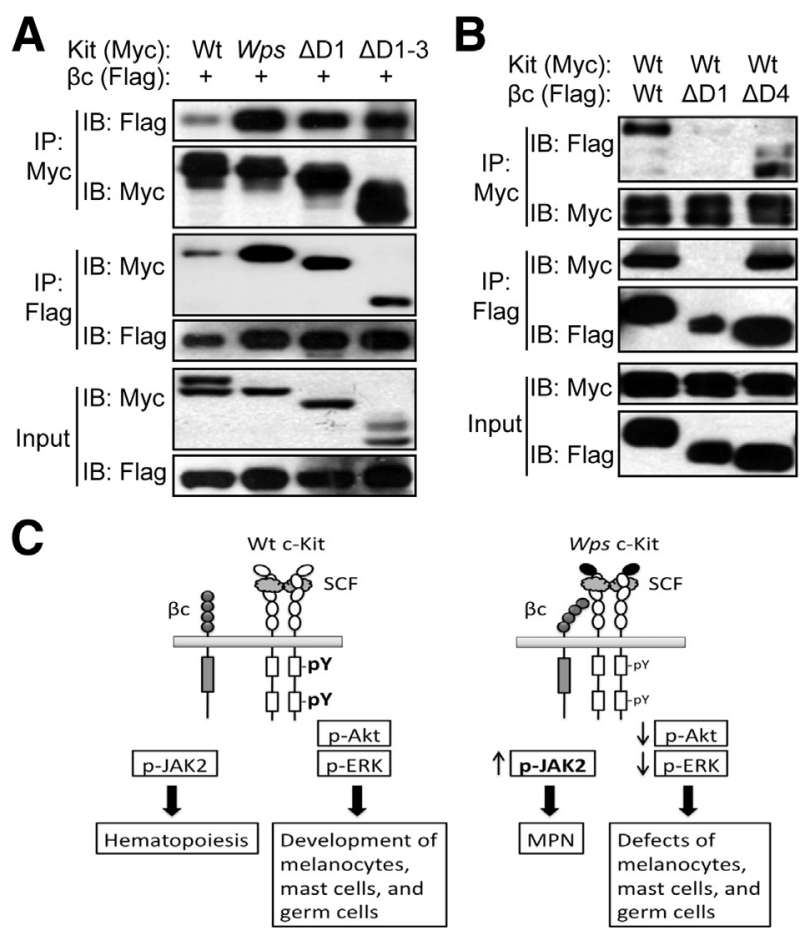

Figure 8 The first domains of Kit and $\beta_{\mathrm{c}}$ receptor are important for their interaction. A: Myc-tagged Wt, Wps, and D1, D1 to D4 deletions of Kit proteins were co-transfected with Flag-tagged $\beta_{\mathrm{c}}$ receptor in HEK 293 cells. Reciprocal co-immunoprecipitations show their interaction. B: Interaction of Kit with Wt, D1, and D4 deletions of $\beta_{c}$ receptor in HEK 293 cells. C: Schematic signaling transduction of Kit. Left: When activated by SCF, Wt Kit autophosphorylates itself and elicits downstream ERK, Akt, and JAK2 activation to mediate its biological functions. Right: With decreased binding to SCF, Wps Kit shows decreased autophosphorylation and ERK and Akt activation, which results in defects in development of melanocytes, mast cells, and germ cells. However, Wps Kit forms heterodimer with $\beta_{c}$ receptor and recruits more JAK2 and activates JAK2, which leads to the myeloproliferation in mice. IB, immunoblotting; IP, immunoprecipitation. 
cooperation with IL-3R $\beta_{\mathrm{c}}$ chain are both responsible for constitutive association and activation of JAK2, although we cannot exclude the possibility that other molecules are also involved. Another potential candidate is Epo- $\mathrm{R}^{19}$; however, we failed to detect any interaction between Kit and Epo-R in our overexpression system (Supplemental Figure S8).

In a study of 25 patients with MPNs, a D52N substitution in KIT was found in one patient with CML and two patients with primary myelofibrosis. ${ }^{42}$ The investigators postulated with no evidence that the D52N mutation affects the SCFbinding or the ligand-induced dimerization of KIT, which would lead to enhancement of the receptor kinase activity. D52 and D60, located at two ends of the B strand of the D1 are conserved in multiple species ${ }^{16,17}$; therefore, Wps mice may mimic the $D 60 N$ mutation in humans. Inokuchi et $\mathrm{al}^{43}$ and Fontalba et $\mathrm{al}^{11}$ have identified KIT mutations in patients with CML and polycythemia vera. However, characterization of these mutations in MPNs is desired. The increase in constitutive recruitment and activation of JAK2 in Wps mice suggests overactivation of JAK2 as one molecular mechanism in human MPNs with KIT mutations.

In conclusion, Wps mice with a $D 60 N$ mutation facilitate our understanding of the D1 of KIT and possibly other receptor tyrosine kinases and serve as an animal model to study myeloproliferation with KIT mutations. Our findings provide insights into the pathogenesis of MPNs and also have important implications for the diagnosis and treatment of MPNs.

\section{Acknowledgments}

We thank Dr. Zhenguo Wu (Hong Kong University of Science and Technology) for HA-tagged mouse JAK2 expression plasmid; Dr. Zhongzhou Yang (Model Animal Research Center) for HA antibody; Drs. Daniel DiMaio and Stephanie Halene (Yale University School of Medicine) for providing HA-mEpo-R plasmid and helpful discussions, respectively; Zai Chang, Yanfeng $\mathrm{Li}$, and Yue Zhou for technical assistance; and Colleen Feriod and Kevin Qian for critical reading of the manuscript.

\section{Supplemental Data}

Supplemental material for this article can be found at http://dx.doi.org/10.1016/j.ajpath.2013.09.019.

\section{References}

1. Tefferi A, Vardiman JW: Classification and diagnosis of myeloproliferative neoplasms: the 2008 World Health Organization criteria and point-of-care diagnostic algorithms. Leukemia 2008, 22:14-22

2. De Keersmaecker K, Cools J: Chronic myeloproliferative disorders: a tyrosine kinase tale. Leukemia 2006, 20:200-205

3. Tefferi A, Gilliland DG: Oncogenes in myeloproliferative disorders. Cell Cycle 2007, 6:550-566

4. Baxter EJ, Scott LM, Campbell PJ, East C, Fourouclas N, Swanton S, Vassiliou GS, Bench AJ, Boyd EM, Curtin N, Scott MA, Erber WN,
Green AR: Acquired mutation of the tyrosine kinase JAK2 in human myeloproliferative disorders. Lancet 2005, 365:1054-1061

5. Scott LM, Tong W, Levine RL, Scott MA, Beer PA, Stratton MR, Futreal PA, Erber WN, McMullin MF, Harrison CN, Warren AJ, Gilliland DG, Lodish HF, Green AR: JAK2 exon 12 mutations in polycythemia vera and idiopathic erythrocytosis. N Engl J Med 2007, 356:459-468

6. Pietra D, Li S, Brisci A, Passamonti F, Rumi E, Theocharides A, Ferrari M, Gisslinger H, Kralovics R, Cremonesi L, Skoda R, Cazzola M: Somatic mutations of JAK2 exon 12 in patients with JAK2 (V617F)negative myeloproliferative disorders. Blood 2008, 111:1686-1689

7. James C, Ugo V, Le Couedic JP, Staerk J, Delhommeau F, Lacout C, Garcon L, Raslova H, Berger R, Bennaceur-Griscelli A, Villeval JL, Constantinescu SN, Casadevall N, Vainchenker W: A unique clonal JAK2 mutation leading to constitutive signalling causes polycythaemia vera. Nature 2005, 434:1144-1148

8. Kralovics R, Passamonti F, Buser AS, Teo SS, Tiedt R, Passweg JR, Tichelli A, Cazzola M, Skoda RC: A gain-of-function mutation of JAK2 in myeloproliferative disorders. N Engl J Med 2005, 352:1779-1790

9. Levine RL, Wadleigh M, Cools J, Ebert BL, Wernig G, Huntly BJ, Boggon TJ, Wlodarska I, Clark JJ, Moore S, Adelsperger J, Koo S, Lee JC, Gabriel S, Mercher T, D'Andrea A, Frohling S, Dohner K, Marynen P, Vandenberghe P, Mesa RA, Tefferi A, Griffin JD, Eck MJ, Sellers WR, Meyerson M, Golub TR, Lee SJ, Gilliland DG: Activating mutation in the tyrosine kinase JAK2 in polycythemia vera, essential thrombocythemia, and myeloid metaplasia with myelofibrosis. Cancer Cell 2005, 7:387-397

10. Lennartsson J, Jelacic T, Linnekin D, Shivakrupa R: Normal and oncogenic forms of the receptor tyrosine kinase kit. Stem Cells 2005, 23:16-43

11. Fontalba A, Agirre X, Real PJ, Richard C, Prosper F, FernandezLuna JL: Identification of c-Kit gene mutations in patients with polycythemia vera. Leuk Res 2006, 30:1325-1326

12. Kimura A, Nakata Y, Katoh O, Hyodo H: c-kit Point mutation in patients with myeloproliferative disorders. Leuk Lymphoma 1997, 25: 281-287

13. Ma P, Mali RS, Martin H, Ramdas B, Sims E, Kapur R: Role of intracellular tyrosines in activating KIT-induced myeloproliferative disease. Leukemia 2012, 26:1499-1506

14. Blechman JM, Lev S, Brizzi MF, Leitner O, Pegoraro L, Givol D, Yarden Y: Soluble c-kit proteins and antireceptor monoclonal antibodies confine the binding site of the stem cell factor. J Biol Chem 1993, 268:4399-4406

15. Lev S, Blechman J, Nishikawa S, Givol D, Yarden Y: Interspecies molecular chimeras of kit help define the binding site of the stem cell factor. Mol Cell Biol 1993, 13:2224-2234

16. Liu H, Chen X, Focia PJ, He X: Structural basis for stem cell factorKIT signaling and activation of class III receptor tyrosine kinases. EMBO J 2007, 26:891-901

17. Yuzawa S, Opatowsky Y, Zhang Z, Mandiyan V, Lax I, Schlessinger J: Structural basis for activation of the receptor tyrosine kinase KIT by stem cell factor. Cell 2007, 130:323-334

18. Roskoski R Jr.: Structure and regulation of Kit protein-tyrosine kinase-the stem cell factor receptor. Biochem Biophys Res Commun 2005, 338:1307-1315

19. Hong L, Ramdas B, Chen J, Harris C, Wojchowski DM, Kapur R: KIT associated intracellular tyrosines play an essential role in EpoR cosignaling. Cell Signal 2008, 20:1513-1520

20. Jahn T, Sindhu S, Gooch S, Seipel P, Lavori P, Leifheit E, Weinberg K: Direct interaction between Kit and the interleukin-7 receptor. Blood 2007, 110:1840-1847

21. Wu H, Klingmuller U, Acurio A, Hsiao JG, Lodish HF: Functional interaction of erythropoietin and stem cell factor receptors is essential for erythroid colony formation. Proc Natl Acad Sci U S A 1997, 94: $1806-1810$

22. Wu H, Klingmuller U, Besmer P, Lodish HF: Interaction of the erythropoietin and stem-cell-factor receptors. Nature 1995, 377:242-246 
23. Brizzi MF, Zini MG, Aronica MG, Blechman JM, Yarden Y, Pegoraro L: Convergence of signaling by interleukin-3, granulocytemacrophage colony-stimulating factor, and mast cell growth factor on JAK2 tyrosine kinase. J Biol Chem 1994, 269:31680-31684

24. McNiece IK, Langley KE, Zsebo KM: Recombinant human stem cell factor synergises with GM-CSF, G-CSF, IL-3 and epo to stimulate human progenitor cells of the myeloid and erythroid lineages. Exp Hematol 1991, 19:226-231

25. Lennartsson J, Shivakrupa R, Linnekin D: Synergistic growth of stem cell factor and granulocyte macrophage colony-stimulating factor involves kinase-dependent and -independent contributions from c-Kit. J Biol Chem 2004, 279:44544-44553

26. He F, Wang Z, Zhao J, Bao J, Ding J, Ruan H, Xie Q, Zhang Z, Gao X: Large-scale screening of disease model through ENU mutagenesis in mice. Chinese Sci Bull 2003, 48:2665-2671

27. Ruan HB, Zhang N, Gao X: Identification of a novel point mutation of mouse proto-oncogene c-kit through N-ethyl-N-nitrosourea mutagenesis. Genetics 2005, 169:819-831

28. Guo XL, Ruan HB, Li Y, Gao X, Li W: Identification of a novel nonsense mutation on the Pax 3 gene in ENU-derived white belly spotting mice and its genetic interaction with c-Kit. Pigment Cell Melanoma Res 2010, 23:252-262

29. Krutzik PO, Nolan GP: Intracellular phospho-protein staining techniques for flow cytometry: monitoring single cell signaling events. Cytometry A 2003, 55:61-70

30. Lennartsson J, Ronnstrand L: Stem cell factor receptor/c-Kit: from basic science to clinical implications. Physiol Rev 2012, 92:1619-1649

31. Forbes SA, Bindal N, Bamford S, Cole C, Kok CY, Beare D, Jia M, Shepherd R, Leung K, Menzies A, Teague JW, Campbell PJ, Stratton MR, Futreal PA: COSMIC: mining complete cancer genomes in the Catalogue of Somatic Mutations in Cancer. Nucleic Acids Res 2011, 39:D945-D950

32. Majumder S, Brown K, Qiu FH, Besmer P: c-kit protein, a transmembrane kinase: identification in tissues and characterization. Mol Cell Biol 1988, 8:4896-4903

33. Kim DS, Hwang ES, Lee JE, Kim SY, Park KC: Sphingosine-1phosphate promotes mouse melanocyte survival via ERK and Akt activation. Cell Signal 2003, 15:919-926
34. Okayama Y, Kawakami T: Development, migration, and survival of mast cells. Immunol Res 2006, 34:97-115

35. De Miguel MP, Cheng L, Holland EC, Federspiel MJ, Donovan PJ: Dissection of the c-Kit signaling pathway in mouse primordial germ cells by retroviral-mediated gene transfer. Proc Natl Acad Sci U S A 2002, 99:10458-10463

36. Weiler SR, Mou S, DeBerry CS, Keller JR, Ruscetti FW, Ferris DK, Longo DL, Linnekin D: JAK2 is associated with the c-kit protooncogene product and is phosphorylated in response to stem cell factor. Blood 1996, 87:3688-3693

37. Blake TJ, Jenkins BJ, D’Andrea RJ, Gonda TJ: Functional cross-talk between cytokine receptors revealed by activating mutations in the extracellular domain of the beta-subunit of the GM-CSF receptor. J Leukoc Biol 2002, 72:1246-1255

38. McCormack MP, Gonda TJ: Myeloproliferative disorder and leukaemia in mice induced by different classes of constitutive mutants of the human IL-3/IL-5/GM-CSF receptor common beta subunit. Oncogene 1999, 18:7190-7199

39. Jiang X, Gurel O, Mendiaz EA, Stearns GW, Clogston CL, Lu HS, Osslund TD, Syed RS, Langley KE, Hendrickson WA: Structure of the active core of human stem cell factor and analysis of binding to its receptor kit. EMBO J 2000, 19:3192-3203

40. Zhang Z, Zhang R, Joachimiak A, Schlessinger J, Kong XP: Crystal structure of human stem cell factor: implication for stem cell factor receptor dimerization and activation. Proc Natl Acad Sci U S A 2000, 97:7732-7737

41. Blechman JM, Lev S, Barg J, Eisenstein M, Vaks B, Vogel Z, Givol D, Yarden Y: The fourth immunoglobulin domain of the stem cell factor receptor couples ligand binding to signal transduction. Cell 1995, 80: $103-113$

42. Nakata Y, Kimura A, Katoh O, Kawaishi K, Hyodo H, Abe K, Kuramoto A, Satow Y: c-kit point mutation of extracellular domain in patients with myeloproliferative disorders. Br J Haematol 1995, 91: 661-663

43. Inokuchi $\mathrm{K}$, Yamaguchi $\mathrm{H}$, Tarusawa $\mathrm{M}$, Futaki $\mathrm{M}$, Hanawa $\mathrm{H}$, Tanosaki S, Dan K: Abnormality of c-kit oncoprotein in certain patients with chronic myelogenous leukemia-potential clinical significance. Leukemia 2002, 16:170-177 